\title{
Dietary compliance in a human intervention study investigating the impact of specific foods on urinary metabolites
}

\author{
N. D. Willis ${ }^{1}$, S. E. Dodds ${ }^{1}$, A. J. Lloyd ${ }^{2}$, L. Xie ${ }^{1}$, E. S. Chambers ${ }^{3}$, I. Garcia-Perez ${ }^{3}$, G. Frost ${ }^{3}$, \\ M. Beckmann ${ }^{2}$, J. Draper ${ }^{2}$ and J. C. Mathers ${ }^{1}$ \\ ${ }^{1}$ Human Nutrition Research Centre, Institute for Ageing and Health, Newcastle University, Campus for Ageing and \\ Vitality, Newcastle-upon-Tyne, NE4 5PL, UK, ${ }^{2}$ Institute of Biological, Environmental and Rural Sciences, Aberystwyth \\ University, Aberystwyth, SY23 3DA, UK and ${ }^{3}$ Nutrition and Dietetic Research Group, Division of Diabetes, \\ Endocrinology and Metabolism, Department of Medicine, Hammersmith Hospital Campus, Imperial College London, \\ London, W12 0NN, UK
}

Current methods for estimating food intake such as food frequency questionnaires are subject to individual bias and are difficult to validate. These limitations mean that it is difficult to assess the habitual diet of individuals ${ }^{(1)}$. However investigation of food-derived metabolites in bio-fluids could provide an alternative objective approach to measurement of food consumption ${ }^{(2)}$. The MAIN (Metabolomics at Aberystwyth, Imperial and Newcastle) Study is using metabolomics-based approaches to discover novel metabolite biomarkers for specific foods. This requires feeding volunteers specific foods over several days with collection of urine and blood at pre-determined times. The present study aimed to measure the levels of compliance and acceptability with such a dietary intervention protocol.

Ten healthy adults ( 5 females) were recruited and provided with all foods and drinks for three days (identical menus for all participants). Acceptability was determined daily using a five part questionnaire which assessed perceptions of convenience, quality, taste, variety and health of the food items using Likert scales ${ }^{(3)}$. Compliance was calculated as a percentage of total items eaten at each meal using both subjective and objective methods.

There was a high level of compliance $(88.4 \%$, SD $7 \cdot 7 \%$ ) with, and acceptability (7.0, SD 0.5 , on a 10 point scale with 10 being maximum) of, the dietary intervention. There was a positive linear correlation between acceptability of the experimental foods and compliance with the study protocol. Participant weight and sex did not affect compliance significantly.
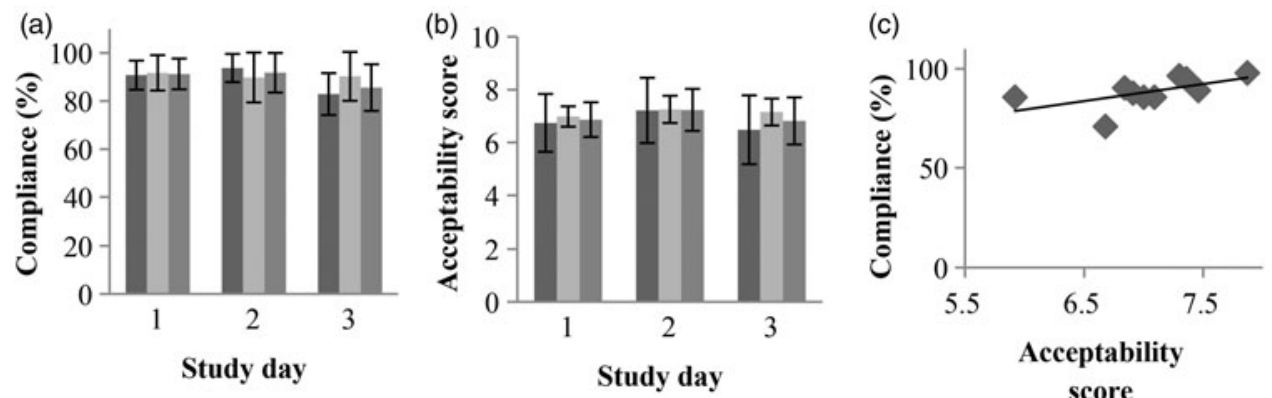

Fig. 1. Compliance (a) and acceptability (b) of the daily menu plans $(\square=$ females; $=$ males; $\square=$ total). Compliance with the protocol correlated positively with acceptability of the foods, $p<0.001$ (c).

The tools developed in this study were valuable in assessing the suitability of a three day dietary intervention protocol and the results obtained show high acceptability and compliance with the protocol.

The MAIN Study is funded by the Medical Research Council (Grant No. MR/J010308/1).

1. Bingham SA, Gill C, Welch A et al. (1994) Br J Nutr 72, 619-643.

2. Penn L, Boeing H, Boushey CJ et al. (2010) Genes Nutr 5, 205-213.

3. Drewnowski A and Eichelsdoerfer P (2009) Public Health Nutr 12, 1621-1628. 